\title{
Need for Problem-Based Learning in Clinical Pharmacy Education in India
}

\author{
Uday Venkat Mateti ${ }^{1}$, Shiv Sagar Konda', M.G. Irfan Ahmed Khan², Anantha Naik Nagappa*1 \\ Manipal College of Pharmaceutical Sciences, Manipal University, Manipal, Karnataka-576104, India \\ St. Peter's Institute of Pharmaceutical Sciences, Warangal, Andhra Pradesh-506002, India.
}

\begin{abstract}
Problem-Based Learning (PBL) is an instructional student centered approach, which serves to enhance skills such as problem-solving, critical thinking, clinical reasoning and self-directed learning to develop a solution to a defined problem. This review serves the need of changes in the teaching methodology in clinical pharmacy education, where the clinical pharmacy students are involved in elaborative patient care responsibility which embodies exclusive communication skills, increased problem solving capabilities, effective critical thinking abilities and sound decision making skills. As an alternative to current traditional or instructive technique of one way of knowledge transformation from teacher to students, a passive approach where the participation of the student in the learning process is restricted. It also creates an environment for student group dynamics in an open PBL setting. In this model a mentor should act as a facilitator rather than an instructor. The PBL incorporates basic elements of learning and integrate theory and practice and relates the basic to the applied knowledge. Furthermore there is a need to validate the effectiveness of PBL with conventional class room teaching. Unless the practical problems are not posed to the students the development of accessible effective knowledge is not achieved. Hence the PBL ensures highest participation of the students. Thus the PBL can be incorporated in the future clinical pharmacy practice curriculum to make PharmD course an effective in training skilled clinical pharmacist.
\end{abstract}

Keywords: Problem Based Learning, India, Pharmacy, Education, PharmD

\section{INTRODUCTION}

Problem-Based Learning (PBL) is an instructional teaching strategy that emphasize on teaching students by using realistic scenarios. It is a student-centered training, where the students collaboratively solve problems and reflect on their experiences in order to learn concepts and retain them for lifelong. ${ }^{1}$ It is a system that recognizes the need to develop problem solving skills as well as the necessity of enlightening students with new knowledge and skills. PBL also serves to develop the processes of the activation and elaboration of prior knowledge, restructuring of knowledge, learning in context and epistemic curiosity. ${ }^{2}$ In comparison to students enrolled in a more traditional curriculum, PBL students might be more highly motivated, better problemsolvers, more able to integrate basic science into clinical problems and more self directed learners. $^{3}$

\section{Necessity of Problem-Based Learning in Indian Pharmacy Education}

Clinical pharmacy is the area where the pharmacist, being a part of the integrated health care team gives pharmaceutical services to the physician and also the patients in hospital. The multidisciplinary healthcare team reflects the responsibility of each of the member from different streams. Clinical pharmacist looking after appropriateness of therapy, has his role in solving various problems in managing pharmacotherapy which
Submitted Date : 19-08-2014 Accepted Date : 04-09-2014

DOI: 10.5530/ijopp.7.3.3

Address for correspondence: Dr. Anantha Naik Nagappa Professor, Department of Pharmacy Management, Manipal College of Pharmaceutical Sciences, Manipal University, Manipal, Karnataka-576104, India.

E-mail: anantha1232000@ gmail.com

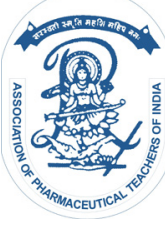

www.ijopp.org 
he comes across in his daily professional life. There is a need to introduce new learning tools like PBL in training the Indian pharmacy students, especially students pursuing pharmacy practice courses like PharmD. The routine classroom teaching is almost a kind of one way communication and is deterrent to the advancement of students' ability of rational thinking and logical reasoning. Problem based learning style is the area where a student decides what type of learning style benefits them the most. So it comes down to the effort that the student puts into their education, let it be PBL or a traditional classroom setting, ultimately determines how much they will retain. Problem Based Learning is the teaching methodology which is proven best in enhancing the knowledge, self direction and motivation besides improving problem solving and decision making skills. ${ }^{4}$ It recommends future directions of group dynamics in a PBL setting to incorporate highest participation of the students on learning and achievement to assess the effects of PBL in Indian Pharmacy Education to make teaching-learning more effective. This teaching methodology should be implemented for PharmD students as special interactive sessions besides regular class room teaching. This should be followed all over the country which could be instrumental in nurturing the budding pharmacists with the agility to work independently and being competent to reach the global standards.

\section{Principles of Problem-Based Learning}

Problems form the focus and they are the initiating variables to a student where he/she can focus with. Meanwhile they develop problem solving skills in finding a solution on their own. Teachers are facilitators/perceptors in this part and they make eight to ten participants in group dynamics and allow the students to put their ideas in resolving the clinical issue. To begin such a kind of study the perceptors set the problems and students attempt to resolve them in parallel by inquiring the preceptors. This involves the team to think and act with motivation seriously as they are made to face the reality of the clinical situation which is more realistic and interactive. Here they are guided by standard protocols of treatment guidelines which make them to understand the significance of the treatment protocols.

\section{Characteristics of Problem-Based Learning}

Problem-based learning is practice oriented in structure with a support from the curriculum and not part of a didactic curriculum. The group members are broken up into four possible roles; they are Project Leader, Facilitator, Recorder, Team member. This is a constructive process in which the perceptors act as providers of problem on which the students suppose to resolve using the established protocols and guidelines. It rein- forces and builds a strong opinion among students who developed a standard approach towards treatment provisions. The obstacle to implement and succeed in this approach is in the formulation of rational treatment approach instead of irrational protocol involving multidisciplinary but contradictory protocols. A preceptor himself is a practicing fellow who guides the learning process and conducts a thorough debriefing at the conclusion of the learning experience. Student's serves as solution providers who attempt to identify the root cause issue and the extent of the severity of the condition which needs a well adequate approach for a good resolution of the clinical issue; this furthers the students to metamorphose into a self-directed learners. ${ }^{5}$

The PBL process as comprises of multiple steps which are critical in solving clinical issues which is unique feature approach not present in other teaching strategies. ${ }^{6}$ The perceptors while screening the case studies identifies a real time clinical issue which could be related to either diagnosis or pharmacotherapy. In case study when real time cases are not possible one may prepare simulated case studies incorporated with clinical issues for learning purpose. There may arise many doubtful conceptions among students, regarding the case study given by the preceptor. Students must interact with their perceptors to have a clarification about the issues. Then students have to define the clinical issue and review the conditions which need more in depth study come explanation. Analysis, brainstorming and creating potential hypothesis is a kind of routine activity which facilitate the students for effective PBL. Perceptors should be available for students to open up discussions for evaluating and organizing possible explanations leading into a potential hypothesis. PBL process has been popular for generating and prioritizing learning objectives and dividing into practical workload which can be objectively investigated which forms the critical aspect of PBL. PBL also gives a provision for private study time to research objectives. During next tutorial, the gained information should be reported back, new information in relation to the problem is synthesized and explained. ${ }^{7}$

\section{Role of Perceptors and Student in PBL}

A Preceptor should be able to create opportunities with real-world, complex and open-ended clinical issues that might be faced in the workplace or daily practice. They must act as facilitators, ensuring the students to get exposed to the challenges which provoke critical thinking. If required they must hoist questions to student groups which can facilitate to deepen the connections that they make among concepts. A student engagement should be focused on the clinical issues along with possible logical resolutions. They should be taught the 


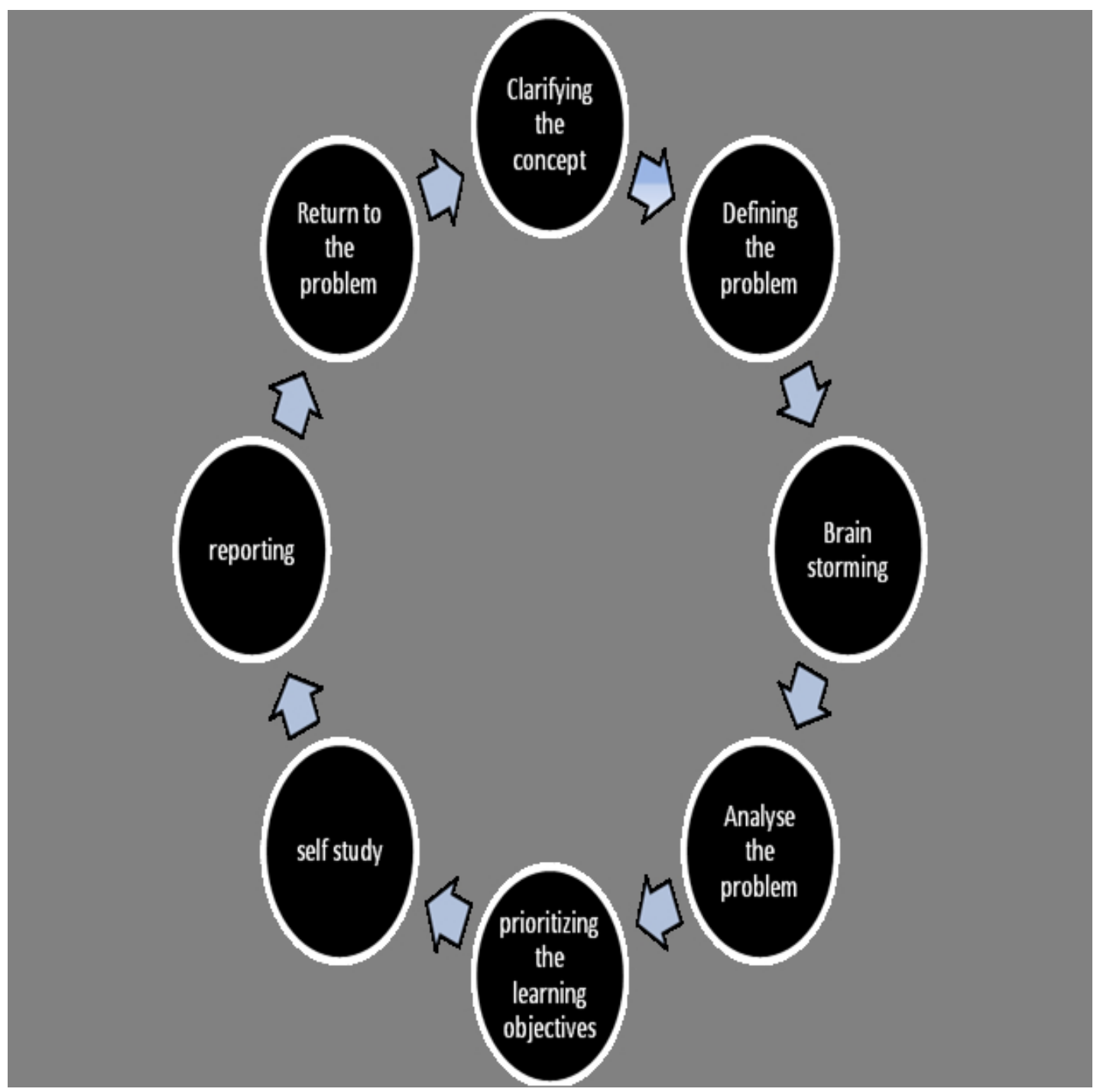

Figure 1: Process of induction of AMC's under PvPI.

skills to collaborate with fellow healthcare professions in order to integrate the clinical resolution of the case study, there should be an open environment in which one can pose questions to preceptor and also cross check a literature and peers to reinforce the practicing skills. ${ }^{5}$ The system of PBL should be able to develop maturity and the decision-making skills in the entire process as they advance their learning.

\section{Benefits of Problem-Based Learning}

As the process of care giving involves simulation or live patients the students are likely to respond to the call of the situation with much seriousness and responsible. Hence one can expect to have longer retention time of knowledge. There are opportunities for mutual consultation, leading to a team work, promoting collaborations with fellow peers, perceptors and other co-healthcare providers. This is an important skill as one should always need to interact and collaborate and should develop sense of inter professional team work, which is a key to the quality care in routine practice. There are prospects to learn from real world problems, which is a unique feature of PBL. It provokes students to use learning and informational resources to a maximum level. ${ }^{8} \mathrm{PBL}$ is found to be instrumental in molding students' ability to use hypothesis driven reasoning in explaining a case. ${ }^{6}$ Besides beneficial to the students, PBL also enhances the subject expertise of the facilitators, as they do stupendous ground work in preparing the simulated cases and related questions. ${ }^{2,9}$ It is also evident from a randomized control trial that the comprehension and depth of knowledge of PBL students is comparatively higher than non-PBL students. ${ }^{10}$

\section{Barriers to Problem-Based Learning}

The major hurdle of PBL is acceptance and implementation by the perceptors who are routinely used to non-PBL method of education. There can be a stiff 
resistance to the implementation, if not explained with adequate training of the perceptors to adopt PBL instead of conventional mode of training. In order to effectively implement PBL, teachers must adopt new roles that are frequently different from those of what went before. In country like India, for many preceptors such a change is unsustainable due to a misconception that the former approaches were good enough to train the students with the skills of pharmaceutical care. However they fail to visualize the changed scenario of the mode and methods of information retrieval and access. Further the application of innovative tools and approaches and their impact on healthcare delivery has caused the gap in knowledge of perceptors. There is a need to conduct various seminars for perceptors to update their knowledge and skills on continuous basis. As the PBL approach is responsive, interactive case study basis it is just not possible to make specific teaching material or notes like in conventional method of training. The acceptance of PBL is slow because many administrators, curriculum developers and teachers lack experience in interdisciplinary education. ${ }^{11}$

\section{CONCLUSION}

Memorizing, reproducing and implementing ideas brought out of the knowledge which is thought in the clinical and academic set up is very essential for any student pursuing healthcare professional course. The traditional teaching method is certainly effective in giving subjective information, but doesn't determine the students' applicability of knowledge gained through lectures. This novel educational approach can be used as an additional learning tool especially in a country like India where the students' role is memorizing and repetition of facts. No two students are same in retaining the information. So there is a need of a proper and standardized method of teaching which could be generalized and would be beneficial to large masses of students. The Purpose behind PBL is to increase the retention of learning and to assess learning in ways, which demonstrate understanding, to develop flexible knowledge, effective collaboration skills and intrinsic motivation to develop a solution to a defined problem.

\section{CONFLICT INTEREST}

Nil

\section{REFERENCES}

1. Bligh J. Problem-based learning in medicine: an introduction. Postgrad Med J. 1995; 71(836): 323-6.

2. Schmidt HG, Vander A, Moust JH, Kokx I, Boon L. Influence of tutors' subject-matter expertise on student effort and achievement in problembased learning. Acad Med. 1993; 68(10): 784-91.

3. Norman GR, Schmidt HG. The psychological basis of problem-based learning: a review of the evidence. Acad Med. 1992; 67(9): 557-65.

4. Cisneros RM, Salisbury-Glennon JD, Anderson-Harper HM. Status of Problem-Based Learning Research in Pharmacy Education: A Call for Future Research. Am J Pharm Educ. 2002; 66(1): 19-26.

5. Akcay B. Problem-Based Learning in Science Education. Journal of Turkish Science Education. 2009; 6(9): 26-36.

6. Hmelo CE, Gotterer GS, Bransford JD. A theory-driven approach to assessing the cognitive effects of PBL. Instructional Science. 1997; 25(6): 387-408.

7. Schmidt HG. Problem-based learning: rationale and description. Med Educ. 1983; 17(1): 11-6.

8. Thomas RE. Problem-based learning: measurable outcomes. Medical Education. 1997; 31(15): 320-9.

9. Janet DW, Cheryl LL. A Review of Problem-based Learning. Journal of Family and Consumer Sciences Education. 2003; 20(1): 16-26.

10. Antepohl W, Herzig S. Problem-based learning versus lecture-based learning in a course of basic pharmacology: a controlled, randomized study. Med Educ. 1999; 33(2): 106-13.

11. Schmidt HG, Moust JH. What makes a tutor effective? A structural-equations modeling approach to learning in problem-based curricula. Acad Med. 1995; 70(8): 708-14. 